\title{
Determination and Isolation of Ginsenosides from Araliaceous Plants by Using Eastern Blotting Fingerprinting
}

Nguyen Huu Tung ${ }^{1}$, Noriko Fukuda², Hiroyuki Tanaka² and Yukihiro Shoyama ${ }^{1 *}$

${ }^{1}$ Faculty of Pharmaceutical Sciences, Nagasaki International University, 2825-7 Huis Ten Bosch, Sasebo, Nagasaki 859-3298, Japan

${ }^{2}$ Faculty of Pharmaceutical Sciences, Kyushu University, 3-1-1 Maidashi, Higashiku, Fukuoka 812-8582, Japan

\begin{abstract}
In the eastern blotting method, a new fashion to separate the ginsenoside molecule into two functional parts using a simple and well-known chemical reaction was developed. In principle, the sugar moieties were oxidized by $\mathrm{NalO}_{4}$ to form dialdehydes, which reacted with amino groups of the protein and covalently bound to the adsorbent PVDF membrane. The MAb bound to the aglycon part of the ginsenoside molecule for immunostaining. Double staining of eastern blotting for ginsenosides using anti-ginsenoside $-\mathrm{Rb}$, and $-\mathrm{Rg}_{1}$ MAbs promoted complete identification of ginsenosides in Panax species. As an application, we analyzed several Araliaceous plants by eastern blotting and enzyme-linked immunosorbent assay (ELISA) using anti-ginsenoside $\mathrm{Rb}_{1}$ MAb leading to the investigation of ginsenoside $\mathrm{Rb}_{1}$ from Kalopanax pictus and Acanthopanax koreanum. On the other hand, by immunoassay-guided fractionation and chromatography separation on the methanol extract of American ginseng, two new minor ginsenosides were isolated. As another application, identification of two known ginsenosides was achieved from the $P$. japonicas extract using eastern blotting and immunoaffinity column combined with anti-ginsenoside Rb, MAb.
\end{abstract}

Keywords: Araliaceae; Acanthopanax koreanum; Kalopanax pictus; Panax quinquefolium; Ginsenoside; Ginsenoside $\mathrm{Rb}_{1}$; Monoclonal antibody; Eastern blotting

\section{Introduction}

Ginseng saponins, commonly called ginsenosides, are the principal components in Panax species and have been occurred in several other Araliaceae plants. According to different aglycones, ginsenosides can be classified into three types: the 20 (S)-protopanaxadiol type such as ginsenosides $R b_{1}, R c, R b_{2}$, and $R d$, the 20 (S)-protopanaxatriol type such as ginsenosides $\mathrm{Rg}_{1}$ and $\mathrm{Re}$, and the oleanolic acid type including ginsenoside Ro and polyacetyleneginsenoside Ro, respectively [1]. These compounds have been widely investigated for their effects on disturbances of the central nervous system, hypothermia and tumor metastasis, and for their antioxidant, antidiabetes, antiaging and radioprotective activities [2]. Especially, ginsenoside $\mathrm{Rb}{ }_{1}$, one of major ginsenosides, exhibited remarkable effects on the central nervous system $[2,3]$ and drug-induced memory impairment [4]. In addition, the regulation of ChAT, NGF and trkA mRNA expression by ginsenoside $\mathrm{Rb}_{1}$ in the rat brain was observed [3]. Therefore, ginsenosides including ginsenoside $\mathrm{Rb}_{1}$ have played as important resources in the development of new drugs [5].

For qualitative and quantitative analyses of ginsenosides, thin layer chromatography (TLC) [6], high performance liquid chromatography (HPLC) [7,8], and liquid chromatography-mass spectrometry (LCMS) [9] have been routinely used. Recently, an enzyme-linked immunosorbent assay (ELISA) system has been opened for natural product analysis as the most promising methodology. We have developed the preparation of monoclonal antibodies (MAbs) against ginsenosides $\mathrm{Rb}_{1}$ [10], $-\operatorname{Rg}_{1}$ [11], and - $\mathrm{Re}$ [12]. Furthermore, we set up the ELISA using individual MAbs and applied for the quantitative analysis of ginsenosides [13,14]. On the other hand, regarding immunostaining we succeeded to immunostain the steroidal alkaloid glycosides using anti-solamargine MAb [15] in the first stage, then established the immunostaining methods for ginsenosides- $R b_{1}$ and $-R g_{1}$ [13]. According to development of new staining method for glycosides, we named this methodology as eastern blotting for immunostaining of glycyrrhizin [16].

\section{Eastern blotting fingerprint for ginsenosides}

The ginsenoside $\mathrm{Rb}_{1}, \mathrm{Rc}, \mathrm{Rd}, \mathrm{Rg}_{1}$ mixture was applied to TLC plates and developed with $n$-BuOH-EtOAc- $\mathrm{H}_{2} \mathrm{O}(15: 1: 4, \mathrm{v} / \mathrm{v} / \mathrm{v})$. One TLC plate developed was sprayed and stained with $\mathrm{H}_{2} \mathrm{SO}_{4}$. Another TLC plate developed was blotted on the PVDF membrane by heating at around $120^{\circ} \mathrm{C}$ for short period, the PVDF membrane was treated with $\mathrm{NaIO}_{4}$ solution to release aldehyde groups in sugar moieties, then reacted with proteins such as bovine serum albumin (BSA) resulting in ginsenoside-BSA conjugates which can fix on the PVDF membrane. On the other hand, an aglycone and a part of sugar moiety as an antigen can be stained by MAb like western blotting. Therefore, it became evident that ginsenosides having small cross-reactivities for anti-ginsenosides $\mathrm{Rb}_{1}$ and anti-ginsenoside $\mathrm{Rg}_{1}$ MAbs could be stained in the case of eastern blotting for ginsenosides, suggesting that the specific reactivity of the sugar moiety in the ginsenoside molecule against MAb might be modified by $\mathrm{NaIO}_{4}$ treatment of the ginsenoside on the membrane, resulting that other ginsenosides having protopanaxatriol as an aglycone such as ginsenosides $\mathrm{Re}, \mathrm{Rf}$ and $\mathrm{Rg}_{3}$ can be detectable by eastern blotting using anti-ginsenoside $\mathrm{Rg}_{1} \mathrm{MAb}$ although $3.3 \%$ of cross-reactivity for ginsenoside Re [13] (Figure 1). Likewise, ginsenosides possessing protopaxadiol as an aglycone like ginsenoside $\mathrm{Rc}, \mathrm{Rd}$ and $\mathrm{Rb}_{2}$ can be stained using anti-ginsenoside $\mathrm{Rb}_{1} \mathrm{MAb}$ [17] (Figure 2). This finding is important for the surveys of saponins having two types of aglycone like ginsenosides.

\section{Analysis of ginsenoside by eastern blotting fingerprinting}

Determination of ginsenoside $\mathrm{Rb}_{1}$ from Acanthopanax koreanum Nakai: Acanthopanax koreanum (Araliaceae), which is a perennial shrub and distributed in Northeast Asia, has been used as a tonic and for treatment of rheumatism, allergies, hepatitis, and diabetes $[18,19]$.

*Corresponding author: Yukihiro Shoyama, Professor, Faculty of Pharmaceutical Sciences, Nagasaki, International University, Nagasaki 859-3298, Japan, Tel: +81956-20-5653; Fax: +81-956-20-5653; E-mail: shoyama@niu.ac.jp

Received March 28, 2013; Accepted April 24, 2013; Published April 30, 2013

Citation: Tung NH, Fukuda N, Tanaka H, Shoyama Y (2013) Determination and Isolation of Ginsenosides from Araliaceous Plants by Using Eastern Blotting Fingerprinting. Nat Prod Chem Res 1: 107. doi:10.4172/2329-6836.1000107

Copyright: (C) 2013 Tung NH, et al. This is an open-access article distributed under the terms of the Creative Commons Attribution License, which permits unrestricted use, distribution, and reproduction in any medium, provided the original author and source are credited. 


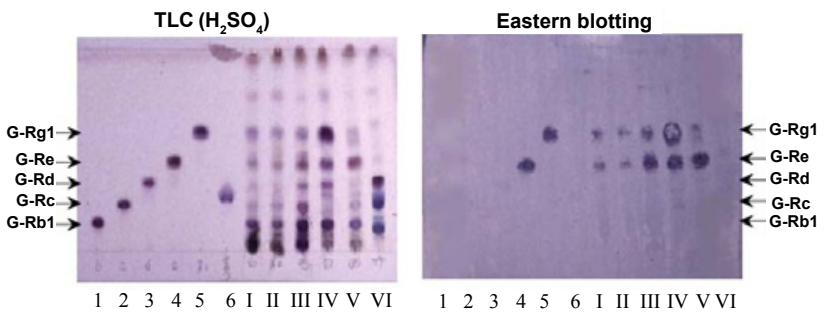

Lanes 1, 2, 3, 4, 5 and 6 indicate ginsenoside $\mathrm{Rb}, \mathrm{Rc}, \mathrm{Rd}, \mathrm{Re}, \mathrm{Rg}$ and chikusetsusaponin IV, respectively. Lanes I, II, III, IV, V and VI are white ginseng, red ginseng, fibrous ginseng, $P$. notoginseseng, $P$. quinquefolium and $P$. japonicus, respectively.

Figure 1: Fingerprinting of ginsenoside standards and ginsenosides in various ginseng by $\mathrm{H}_{2} \mathrm{SO}_{4}$ (left) and eastern blotting (right) using anti-ginsenoside $\mathrm{Rg}$, monoclonal antibody.
A. $\mathrm{TLC}\left(\mathrm{H}_{2} \mathrm{SO}_{4}\right)$

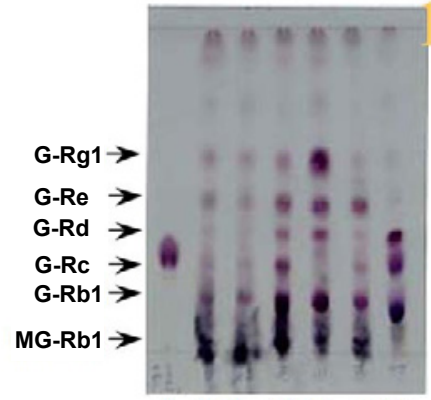

1 I II III IV V VI

\section{B. Eastern blot}

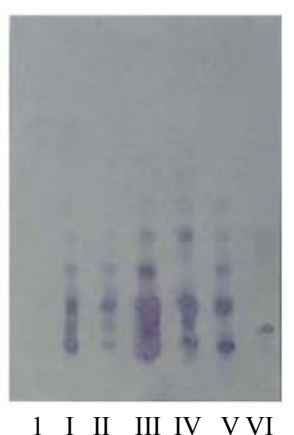

Lanes 1, chikusesusaponin IV; II, III, IV, V and VI indicate white ginseng, red ginseng, fibrous ginseng, $P$. notoginseseng, $P$. quinquefolium and $P$. japonicus, respectively.

Figure 2: Fingerprinting of ginsenosides in various ginseng by $\mathrm{H}_{2} \mathrm{SO}_{4}$ (left) and eastern blotting (right) using anti-ginsenoside $\mathrm{Rb}_{1}$ monoclonal antibody.

Bioactive constituents of $A$. koreanum have been reported including several lignans, diterpenes [20,21], and lupane-type triterpene glycosides, which are considered as major constituents [22,23]. Since we have established the combination of ELISA and eastern blotting methods using anti-ginsenoside $\mathrm{Rb}_{1} \mathrm{MAb}$ for the identification of ginsenoside $\mathrm{Rb}_{1}$ in Panax species and traditional Chinese medicines [14] as a high sensitive and rapid method, the finding and determination of ginsenoside $\mathrm{Rb}_{1}$ in $A$. koreanum of Araliaceae family by using ELISA and eastern blotting monitoring will be reviewed in this section.

Figure 3 showed the double eastern blotting staining (A) and the $\mathrm{H}_{2} \mathrm{SO}_{4}$ staining (B) profiles of ginsenoside standards (right) and crude extracts of $A$. koreanum (left) using anti-ginsenoside $\mathrm{Rb}_{1}$ and antiginsenoside $\mathrm{Rg}_{1}$ MAbs. Although $\mathrm{H}_{2} \mathrm{SO}_{4}$ staining detected clearly all standard ginsenosides without color differences, the TLC profile of $A$. koreanum crude extract revealed complicated fingerprinting patterns indicating that ginsenosides are ambiguously determined. From double eastern blotting we confirmed that no ginsenoside having protopanaxatriol as an aglycone was contained in the A. koreanum crude extract because no purple spot appeared. On the other hand, blue spots were detected meaning that protopanaxadiol type ginsenosides are contained in A. koreanum. It is clear that Rf value on TLC reflects the sugar number in general. From these evidences it is easily suggested that $A$. koreanum contains small amount of ginsenoside $\mathrm{Rb}_{1}$ and a more

polar ginsenoside which cannot be longer analyzed due to its trace amount [24].

We analyzed A. koreanum leaves crude extract by competitive ELISA using anti-ginsenoside $\mathrm{Rb}_{1} \mathrm{MAb}$ in order to confirm the existence and concentration of ginsenoside $\mathrm{Rb}_{1}$ resulting in $0.000016 \%$ dry wt. of ginsenoside $\mathrm{Rb}_{1}$. The roots and stems were also analyzed separately by the same manner finding concentrations of $0.000039 \%$ and $0.000014 \%$ dry wt., respectively. The concentrations of ginsenoside $\mathrm{Rb}_{1}$ in the sample is extremely low, therefore, it has been suggested that chromatographic purification and analyses of ginsenoside $\mathrm{Rb}_{1}$ have been unaffordable to date. To our knowledge, this is the first evidence of ginsenoside $\mathrm{Rb}_{1}$ in Acanthopanax species [24]. The results further support potential and promising application of MAb such as eastern blotting and ELISA for surveying ginsenoside sources.

Determination of ginsenosides from Panax japonicas: $P$. japonicas, which is wildly distributed in Japan and China, is morphologically different from the other Panax species. Regarding its constituents, Yahara et al. reported that no ginsenoside $\mathrm{Rb}_{1}$ was found and isolated several dammarane-type saponins structurally related to ginsenoside like chikusetusaponin I VI and oleanane-type saponins named as chikusetsusaponins as major components [25]. In addition, Morita et al. examined the varieties of $P$. japonicus by chemical analysis of saponins [26]. From these results, the concentration of ginsenoside $\mathrm{Rb}_{1}$ might be at trace levels. However, higher concentration compared with previous reports was determined by ELISA [13], although relatively half the concentration of ginsenoside $R b_{1}$ was detected by HPLC analysis compared with ELISA. In order to confirm these differences, immunoaffinity column chromatography was employed for immunoaffinity concentration of ginsenoside $\mathrm{Rb}_{1}$. The crude root extract of $P$. japonicus was subjected to the immunoaffinity column and first washed with the washing solvent (PBS; fraction 1) and then with elution solvent ( $\mathrm{AcOH}$ buffer and $20 \% \mathrm{MeOH}$ (fraction 2) [14].

Figure 4 shows the $\mathrm{H}_{2} \mathrm{SO}_{4}$ staining (A) and the eastern blotting (B) profiles of the two fractions separated by the immunoaffinity

A

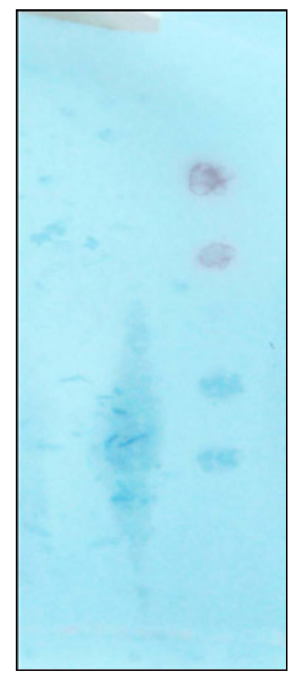

Purple spots are protopanaxtriol saponins and blue spots are protopanaxdiol saponins.

Figure 3: Fingerprinting of ginsenoside standards and crude extract of Acanthopanax koreanum by $\mathrm{H}_{2} \mathrm{SO}_{4}$ (right) and eastern blotting (left) using antiginsenoside $\mathrm{Rb}_{1}$ and $\mathrm{Rg}_{1}$ monoclonal antibodies. 
A

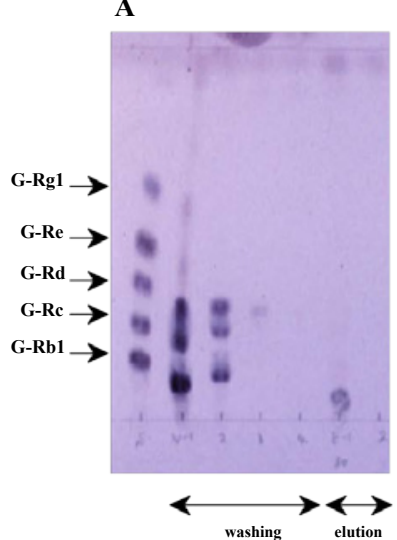

B

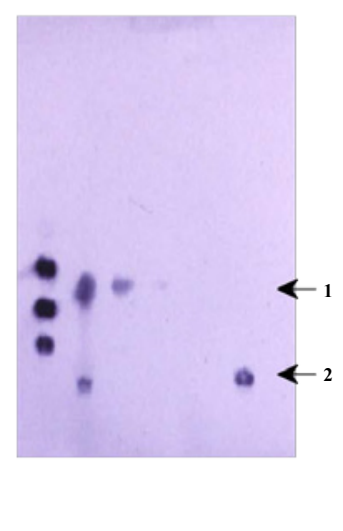

A: stained by $\mathrm{H}_{2} \mathrm{SO}, \mathrm{B}$ : eastern blotting by anti-ginsenoside $\mathrm{Rb}$, washing: fraction 1 elution: fraction 2, compound 1: chikusetsusaponin III, compound 2: chikusetsusaponin VI

Figure 4: Purification and determination of ginsenosides of $P$. japonicas by immunoaffinity column and eastern blotting.

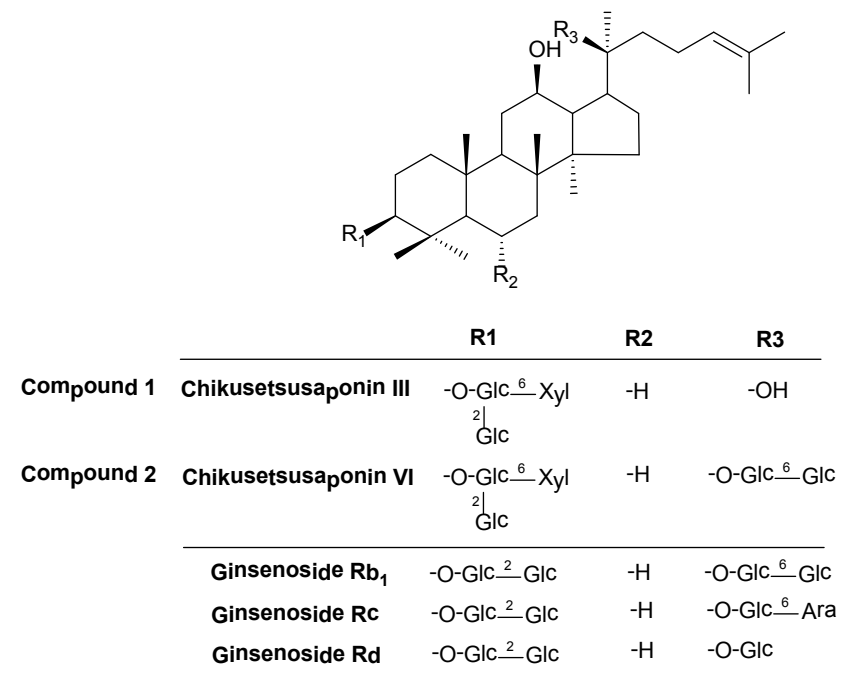

Figure 5: Identified compound 1 and 2 by using immunoaffinity column and eastern blotting and their structures together with ginsenosides $R b_{1}, R c$ and $R d$ in Panax japonicas.

column. Fraction 1 eluted with the washing solvent showed two spots in the eastern blotting profile. A clear unknown spot appeared in fraction 2 eluted with the elution solvent. However, ginsenoside $\mathrm{Rb}_{1}$ was not detected by eastern blotting although it was determined by TLC as indicated in (Figure 4). The unknown compound is proposed to resemble molecular structure and a similar cross-reaction with ginsenoside $R b_{1}$, and likely a ginseng saponin having an aglycone of panaxadiol. Moreover, this compound might have the same sugar fragments in the molecule, but possess an additional sugar moiety compared with ginsenodise $\mathrm{Rb}_{1}$, as indicated by their $R_{f}$ values. These evidences revealed that an unknown compound contained in fraction 2 is chikusetsusaponin VI comparing with the authentic sample directly (Figure 5).

The eastern blotting fingerprinting of fraction 1 indicated that an unknown ginsenoside has protopanaxadiol as an aglycone and three sugar components compared to the $R_{f}$ value of ginsenoside Rc. Therefore, we determined that the unknown compound contained in fraction 1 is chikusetsusaponin III compared with the authentic sample (Figure 5). From this investigation we confirmed that the saponins having protopanaxdiol as an aglycone can be separated by the immunoaffinity column conjugated with anti-ginsenoside $\mathrm{Rb}_{1} \mathrm{MAb}$. Moreover, we concluded that the combination of immunoaffinity concentration and ELISA is suitable for samples containing lower concentrations of ginsenoside $\mathrm{Rb}_{1}$ since the immunoaffinity column can concentrate the ginsenoside $\mathrm{Rb}{ }_{1}$ concentration [14].

\section{Isolation of ginsenosides by using eastern blotting fingerprinting}

Isolation of ginsenoside $\mathbf{R} \mathbf{b}_{1}$ from Kalopanax pictus Nakai: A number of Araliaceae species, including Panax species, have been used as tonics in Asian folk medicine. On the basis of phytochemical study, Araliaceous plants have been documented to contain similar constituents such as ginsenoside, depending on their chemotaxonomical classification. Various analytical methods have been used to analyze ginsenosides, and of them, ELISA appears to be the most promising methodology, considerably. This section reviewed the distribution of ginsenosides in Araliaceous species and isolation of ginsenoside $\mathrm{Rb}$ from the bark of Kalopanax pictus using ELISA and eastern blotting monitoring. Since the bark of K. pictus (Figure 6 line 13) indicated a positive band, the crude extract was analyzed by competitive ELISA using anti-ginsenoside $\mathrm{Rb}_{1} \mathrm{MAb}$, resulting in $0.0009 \%$ dry wt. of ginsenoside $R b_{1}$. The crude extract was subjected to repeated silica gel column chromatography guided with eastern blotting using anti-ginsenoside $\mathrm{Rb}_{1} \mathrm{MAb}$ to afford compound 1 . Compound 1 was consistent with that of ginsenoside $\mathrm{Rb}$ by eastern blotting, and the physical and spectroscopic data $\left({ }^{1} \mathrm{H}-\right.$ and $\left.{ }^{13} \mathrm{C}-\mathrm{NMR}\right)$ of compound 1 resembled to those of authentic ginsenoside $\mathrm{Rb}_{1}$. Further analyses of the stem bark and leaves by competitive ELISA revealed that a higher concentration of ginsenoside $\mathrm{Rb}_{1}$ was occurred in the leaves $(0.0037 \%$ dr. wt.) than in the bark (0.0009\% dr. wt.) [27]. To our knowledge, this is the first isolation of ginsenoside $\mathrm{Rb}_{1}$ from $K$. pictus although the isolation of various oleanane saponins was reported $[28,29]$. These findings suggested that $K$. pictus might be a new resource of ginsenoside $\mathrm{Rb}_{1}$, and moreover the immunoaffinity column conjugated with antiginsenoside $\mathrm{Rb}_{1}$ MAb [13] is readily applicable for purification of ginsenoside $R b_{1}$ in the final stage of the separation process.

Isolation of new ginsenosides from Panax quenquefolium: American ginseng (Panax quinquefolium L.), which is mainly cultivated in the USA, Canada and China, has been widely used as a tonic and functional foods in various forms such as decoction, powder, tea, capsule, etc. like Asian ginseng (P. ginseng, CA. Meyer). These conventional ginseng products have been reported to have a wide array of pharmacological and physiological actions including antiaging, antidiabetic, anticarcinogenic, analgesic, antipyretic, antistress, and antifatigue, respectively [30-33]. The dammarane-type saponins (ginsenosides) are the major active constituents in American ginseng and with the development of modern chromatography, there are more than 30 ginsenosides such as ginsenosides $\mathrm{Rb}_{1}, \mathrm{Rb}_{2}, \mathrm{Rg}_{1}, \mathrm{Rc}, \mathrm{Rd}$, and $\mathrm{Re}$ reported [34,35]. However, in comparison with a number of researches on Asian ginseng, the study on American ginseng and its constituents is much less extensive.

Figure 7 showed the double eastern blotting staining (A) and $\mathrm{H}_{2} \mathrm{SO}_{4}$ staining (B) profiles of crude extracts of Panax species including American ginseng (line 5) using anti-ginsenoside $\mathrm{Rb}_{1}$ and anti-ginsenoside $\mathrm{Rg}_{1}$ MAbs. In general, since $\mathrm{H}_{2} \mathrm{SO}_{4}$ staining detected all compounds, the TLC profile stained by this reagent showed complicated fingerprinting patterns so that ginsenosides are 

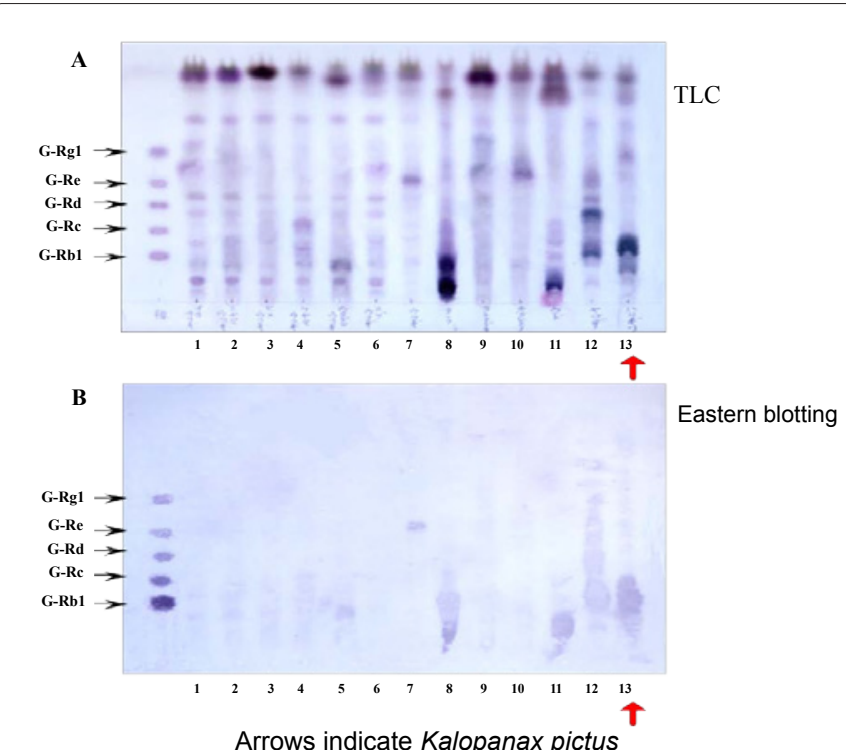

Figure 6: Detection of Ginsenosides in Alariaceae plants detected by $\mathrm{H}_{2} \mathrm{SO}_{4}(\mathrm{~A})$ and eastern blotting $(B)$

ambiguously indicated. On the contrary, eastern blotting obviously and selectively revealed positive spots regarding ginsenosides. The crude extracts of American ginseng clearly indicated ginsenosides $\mathrm{Rg}_{1}, \mathrm{Re}, \mathrm{Rd}, \mathrm{Rc}$ and $\mathrm{Rb}_{1}$ as major ginsenosides. Besides, there have minor spots with the similar color indicating lower $R_{f}$ value than ginsenoside $\mathrm{Rc}$ and ginsenoside $\mathrm{Rb}_{1}$ (Figure 7-A-line 5) in respect to ginsenoside $\mathrm{Rb}_{1}$ (blue) or ginsenoside $\mathrm{Rg}_{1}$ (purple), suggesting more polar ginsenosides. Accordingly, two minor dammarane-type saponins, namely quinquenosides Ja (1) and $\mathrm{Jb}(2)$, were isolated from the American ginseng extract for the first time by immunoassayguided fractionation and chromatography separation (Figure 8). Their structures were elucidated as 6 - $O$ - $[\alpha$-L-rhamnopyranosyl $(1 \rightarrow 2)$ $\beta$-D-glucopyranosyl]-20-O-[ $\beta$-D-glucopyranosyl $(1 \rightarrow 4)-\beta$-Dglucopyranosyl]-3 $\beta, 6 \alpha, 12 \beta, 20 \beta$-tetrahydroxydammar-24-ene and $3-O-[\beta-\mathrm{D}$ - glucopyranosyl $(1 \rightarrow 2)-\beta$-D-glucopyranosyl $]-20$ $O-\{[\alpha$-L-arabinofuranosyl $(1 \rightarrow 6)-\beta$-D-glucopyranosyl $](1 \rightarrow 6)-\beta$-D glucopyranosyl $\}-3 \beta, 12 \beta, 20 \beta$-trihydroxydammar-24-ene (2) on the basis of chemical and spectroscopic methods [36]. The results further supported potential and promising application of MAb such as eastern blotting for surveying new ginsenoside sources.

\section{Conclusions}

It is well known that ginsenosides possesses wide pharmacological activities, and one of them, ginsenoside $\mathrm{Rg}_{3}$ is now an anti-cancer drug in China suggesting that the other ginsenosides may have the possibility of drug development. However, since such ginsenosides can be supplied from ginsengs resulting in high cost performance of ginsenosides, a new supplement system of ginsenosides is needed. From these aims in this review the eastern blotting using anti-ginsenoside $\mathrm{Rb}_{1}$ and/or anti-ginsenoside $\mathrm{Rg}_{1}$ MAbs was applied to analyze the distribution of ginsenosides in Araliaceous plants, like A. koreanum and K. pictus. From $A$. koreanum we determined ginsenoside $\mathrm{Rb}$ by ELISA and eastern blotting using anti-ginsenoside $\mathrm{Rb}_{1}$ MAb. P. quinquefolium were tested, and the structure elucidations of two new ginsenosides from $P$. quinquefolium were succeeded. The combination of eastern blotting and immunoaffinity column conjugated with anti-ginsenoside $\mathrm{Rb}_{1}$ MAb makes it evident to separate ginsenosides having small crossreactivity with anti-ginsenoside $\mathrm{Rb}_{1}$ MAb. In the case of $P$. japonicus we

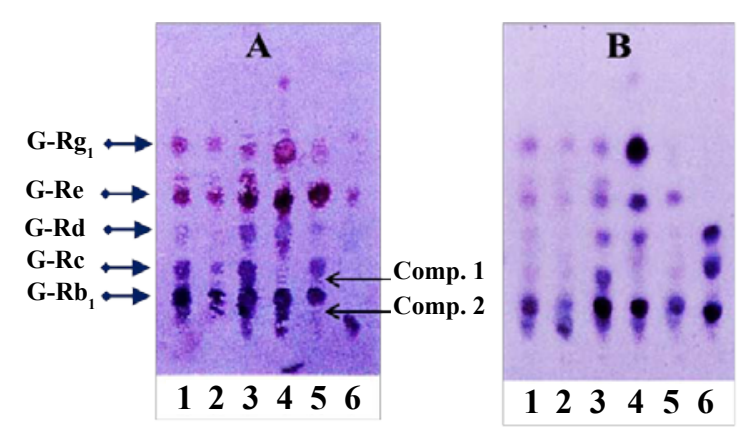

Lanes 1, 2, 3, 4, 5, and 6 are white ginseng, red ginseng, fibrous ginseng, $P$. notoginseseng, $P$. quinquefolium and $P$. japonicus, respectively. Compound 1 and 2 are new ginsenosides isolated from $P$. quinquefolium

Figure 7: Staining of ginsenosides in Panax species by eastern blotting $(A)$ and $\mathrm{H}_{2} \mathrm{SO}_{4}(\mathrm{~B})$.

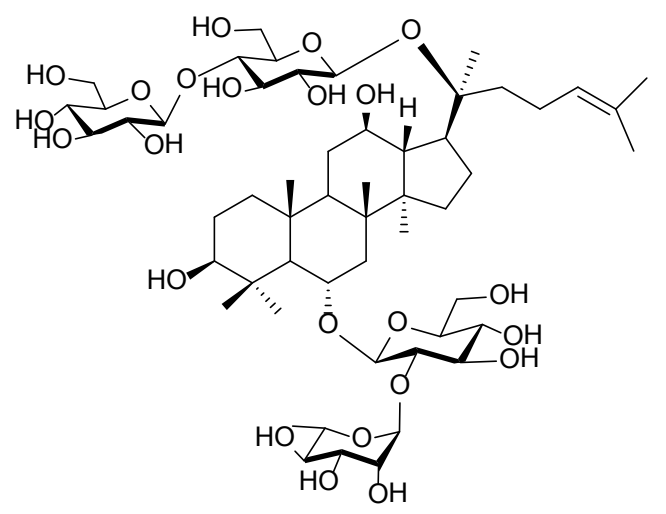

Quinquenoside Ja (1)

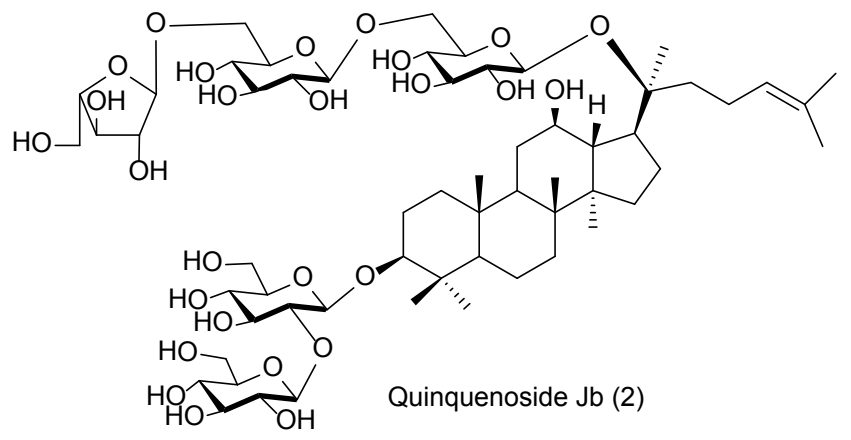

Figure 8: Structures of the two new ginsenosides from $P$. quinquefolium.

used the immunoaffinity column conjugated with anti-ginsenoside $\mathrm{Rb}_{1}$ and separated ginsenosides having the same aglycone with ginsenoside $\mathrm{Rb}_{1}$, and finally determined two ginsenosides. This biotechnological system has a great possibility to widely apply for quick separation of ginsenosides. In fact we succeeded to separate all solasodine glycosides in Solanum khasianum fruits by once immunoaffinity column conjugated with anti-solamargine MAb [37]. The results further 
Citation: Tung NH, Fukuda N, Tanaka H, Shoyama Y (2013) Determination and Isolation of Ginsenosides from Araliaceous Plants by Using Eastern Blotting Fingerprinting. Nat Prod Chem Res 1: 107. doi:10.4172/2329-6836.1000107

Page 5 of 5

supported potential and promising application of MAb such as eastern blotting for surveying pharmacologically active resources.

\section{Acknowledgements}

One of the authors (N.H.T) is grateful to the Japan Society for the Promotion of Science (JSPS) for a Postdoctoral Research Fellowship (P10423) at Nagasak International University.

\section{References}

1. Liu CX, Xiao PG (1990) Recent advances on ginseng research in China. J Ethnopharmacol 36: 27-38.

2. Chang YS, Wu CR, Ho YL, Hsieh MT (1998) In Advances in Ginseng Research Huh H (eds) The Korean Society of Ginseng: Seoul 289-299.

3. Saito H (1989) In Recent Advances in Ginseng Studies, Shibata S, Otsuka Y, Saito H (eds) Hirokawa Publishing Company: Tokyo 99-111.

4. Xie JT, Aung HH, Wu JA, Attele AS, Yuan CS (2002) Effects of American ginseng berry extract on blood glucose levels in ob/ob mice. Am J Chin Med 30: 187-194.

5. Attele AS, Wu JA, Yuan CS (1999) Ginseng pharmacology: multiple constituents and multiple action. Biochem Pharmacol 58: 1685-1693.

6. Kitagawa I, Taniyama T, Shibuya H, Noda T, Yoshikawa M (1987) Chemica studies on crude drug processing. $\mathrm{V}$. On the constituents of ginseng radix rubra (2): comparison of the constituents of white ginseng and red ginseng prepared from the same Panax ginseng root. Yakugaku Zasshi 107: 495-505.

7. Chan TWD, But PPH, Cheng SW, Kwok IMY, Lau FW, et al. (2000) Differentiation and authentication of Panax ginseng, Panax quinquefolius, and ginseng products by using HPLC/MS. Anal Chem 72: 1281-1287.

8. Yamaguchi H, Kasai R, Matsuura H, Tanaka O, Fuwa T (1988) Highperformance liquid chromatography analysis of acidic saponins of ginseng and related plants. Chem Pharm Bull 36: 3468-3473.

9. Wang X, Sakuma T, Asafu-Adjaya E, Shiu GK (1999) Determination of ginsenosides in plant extracts from Panax ginseng and Panax quinquefolius $\mathrm{L}$. by LC/MS/MS. Anal Chem 71: 1579-1584.

10. Tanaka H, Fukuda N, Shoyama Y (1999) Formation of monoclonal antibody against a major ginseng component, ginsenoside $\mathrm{Rb}_{1}$ and its characterization. Cytotechnology 29: 115-120.

11. Fukuda N, Tanaka H, Shoyama Y (2000) Formation of monoclonal antibody against a major ginseng component, ginsenoside $\mathrm{Rg}_{1}$ and its characterization. Monoclonal antibody for a ginseng saponin. Cytotechnology 34: 197-204.

12. Morinaga O, Takuhiro U, Yuan CS, Tanaka H, Shoyama Y (2010) Evaluation of a new eastern blotting technique for the analysis of ginsenoside Re in American ginseng berry pulp extracts. Fitoterapia 81: 284-288.

13. Fukuda N, Tanaka H, Shoyama Y (2000) Application of ELISA, Western blotting and immunoaffinity concentration for survey of ginsenosides in crude drugs of Panax species and traditional Chinese herbal medicines. Analyst 125: 14251429.

14. Tanaka H, Kukuda N, Shoyama $Y$ (2007) Eastern blotting and immunoaffinity concentration using monoclonal antibody for ginseng saponins in the field of traditional Chinese medicines. J Agric Food Chem 55: 3783-3787.

15. Tanaka H, Putalun W, Tsuzaki C, Shoyama Y (1997) A simple determination of steroidal alkaloid glycosides by thin-laer chromatography immunostaining using monoclonal antibody against solamargine. FEBS Lett 404: 279-282.

16. Shan S, Tanaka H, Shoyama Y (2001) Enzyme-linked immunosorbent assay for glycyrrhizin using anti-glycyrrhizin monoclonal antibody and an eastern blotting technique for glucuronides of glycyrrhetic acid. Anal Chem 73: 5784-5790.
7. Tanaka H, Fukuda N, Shoyama Y (2006) Identification and differentiation of Panax species using rapid ELISA and eastern blotting. Phytochem Anal 17: 46-55

18. Lee YN (1996) New Flora of Korea. nhbs 1237

19. Richard ES (1980) Medicinal plants of East and Southeast Asia: Attributed properties. 34: 361

20. Cai XF, Shen G, Dat NT, Kang OH, Kim JA, et al. (2003) Inhibitory effect of TNF- $\alpha$ and IL-8 secretion by pimarane type dirterpenoids from Acanthopanax koreanum. Chem Pharm Bull 51: 605-607.

21. Cai XF, Lee IS, Dat NT, Shen G, Kang JS, et al. (2004) Inhibitory lignans against NFAT transcription factor from Acanthopanax koreanum. Arch Pharm Res 27: 738-741.

22. Choi HS, Kim HJ, Nam SG, Kim IS, Lee KT, et al. (2008) Lupane glycosides from the leaves of Acanthopanax koreanum. Chem Pharm Bull 56: 1613-1616.

23. Nhiem NX, Tung NH, Kiem PV, Minh CV, Ding Y, et al. (2009) Lupane triterpene glycosides from leaves of Acanthopanax koreanum and their cytotoxic activity. Chem Pharm Bull 57: 986-989.

24. Tung NH, Morinaga O, Uto T, Isoda S, Shoyama $Y$ (2013) Analysis of Ginsenoside $\mathrm{Rb}$, from Acanthopanax koreanum by Eastern Blotting and ELISA Using Anti-ginsenoside $\mathrm{Rb}_{1}$ Monoclonal Antibody. Nat Prod Sci, in press.

25. Zou K, Zhu S, Meselhy MR, Tohda C, Cai S, et al. (2002) Dammarane-type Saponins from Panax japonicus and their neurite outgrowth activity in SK-N-SH cells. J Nat Prod 65: 1288-1292.

26. Morita T, Tanaka O, Kohda H (1985) Saponin composition of rhizomes of Panax japonicus collected in South Kyushu, Japan and its significance in oriental traditional medicine. Chem Pharm Bull 33: 3852-3858.

27. Tanaka H, Fukuda N, Shoyama $Y$ (2005) Isolation of ginsenoside $R b_{1}$ from Kalopanax pictus by Eastern blotting using anti-ginsenoside $\mathrm{Rb}$, monoclonal antibody. Phytother Res 19: 255-258.

28. Yao HK, Duan JY, Li Y, Wang JH, Yin XX, et al. (1992) Studies on the chemica constituents of Kalopanax septemlobus. Zhong Yao Cai 34: 716-718.

29. Shao CJ, Kasai R, Ohtani K, Xu JD, Tanaka O (1989) Saponins from leaves of Kalopanax septemlobus (Thunb) Koidz-Structures of kalopanax-saponins La, Lb and Lc. Chem Pharm Bull 37: 3251-3254

30. Court WE (2000) Ginseng: the history of an insignificant plant. Pharm His (Lond) 30: 38-44.

31. Jin Y, Hofseth AB, Cui XL, Windust AJ, Poudyal D, et al (2010) American ginseng suppresses colitis through p53-mediated apoptosis of inflammatory cells. Cancer Prev Res 3: 339-347.

32. Li BH, Wang CZ, He TC, Yuan CS, Du W (2010) Antioxidants potentiate American ginseng-induced killing of colorectal cancer cells. Cancer Lett 289 : 62-70.

33. Yuan CS, Dey $L$ (2001) Multiple effects of American ginseng in clinical medicine. Am J Chin Med 29: 567-569.

34. Qi LW, Wang CZ, Yuan CS (2011) Ginsenosides from American ginseng: Chemical and pharmacological diversity. Phytochemistry 72: 689-699.

35. Yuan CS, Wang CZ, Wicks SM, Qi LW (2010) Chemical and pharmacological studies of saponins with a focus on American ginseng. J Ginseng Res 34: 160167.

36. Tung NH, Shoyama Y (2012) Eastern blotting analysis and isolation of two new dammarane-type saponins from American ginseng. Chem Pharm Bull 60: 1329-1333.

37. Putalun W, Tanaka H, Shoyama Y (1999) Rapid separation of solasodine glycosides by an immunoaffinity column using anti-solamargine monoclonal antibody. Cytotechnology 31: 151-156. 